\title{
WATER WAVES OVER A CHANNEL OF INFINITE DEPTH $\dagger$
}

\author{
By \\ THOM R. GREENE (The General Electric Co., Schenectady, N. Y.) \\ AND ALBERT E. HEINS (Carnegie Institute of Technology)
}

I. Introduction. This is a continuation of some work on gravity waves in channels of finite depth with special types of obstacles (1), (2), (3). Thus far three cases have been considered: the channel of finite depth with (i) a dock; (ii) a submerged plane barrier and (iii) the joining of two free surfaces. In this paper and one to follow we propose to discuss the corresponding problems for channels of infinite depth. The problem which concerns us here is case (ii) and its limiting case (i) for such channels.

The physical background for the problem has been discussed in (1) and (4). Here we shall reformulate the problem so that the notion of infinite depth is admitted. As we have done in the past, only propagating regions will be considered here, the possibility of non-propagating regions may be treated in a fashion similar to that of the propagating region. It will be assumed that the waves have propagation normals which are not perpendicular to the edge of the barrier and in the beginning we shall assume that the barrier is submerged (2). As limiting cases, we will get the case of normal incidence and the "dock problem."

While there is a certain parallelism between the formulation of the problem in channels of infinite and finite depth, we observe that the mathematical methods of solution become more subtle in the channels of infinite depth. Still, we provide here methods which will solve the problem we have described. With minor modifications in method but definite changes in formulation, we can solve (iii) for infinite channels.

II. Fundamental Equations of Motion and Statement of Problem. If we use the linearized equations of motion as we have done in the past, we have for an incompressible, non-viscous fluid, in irrotational motion that

$$
\phi_{x x}^{\prime}+\phi_{y y}^{\prime}+\phi_{z z}^{\prime}=0
$$

where $\phi^{\prime}$ is the velocity potential. On a rigid surface $\phi_{n}^{\prime}=0$, while on a "free surface"

$$
g \phi_{n}^{\prime}+\phi_{t \imath}^{\prime}=0 \text {. }
$$

Assuming monchromatic time dependence

$$
\phi^{\prime}=\phi(x, y, z) \exp (-i \omega t)
$$

and these conditions become $\phi_{n}=0$ and $g \phi_{n}=\omega^{2} \phi$ respectively.

Here we are concerned with a channel of infinite depth with a semi-infinite plane barrier submerged $a$ units below the undisturbed free surface (See Figure 1). We shall assume that wave motion exists for $|x| \rightarrow \infty, y=0$ where the propagation normal is parallel to the free surface but not at right angles to the edge of the barrier, that is the $z$ axis. As such there will be a $z$ variation $\exp (i k z)(k>0)$ in $\phi$ which we may suppress

†Recejved May 22, 1952. This paper is based on research conducted in part under a contract between the Office of Ordnance Research of the Department of the Army and Carnegie Institute of Technology. The paper was submitted by T. R. Greene in partial fulfillment of the requirements for the degree of Doctor of Science at the Carnegie Institute of Technology. 


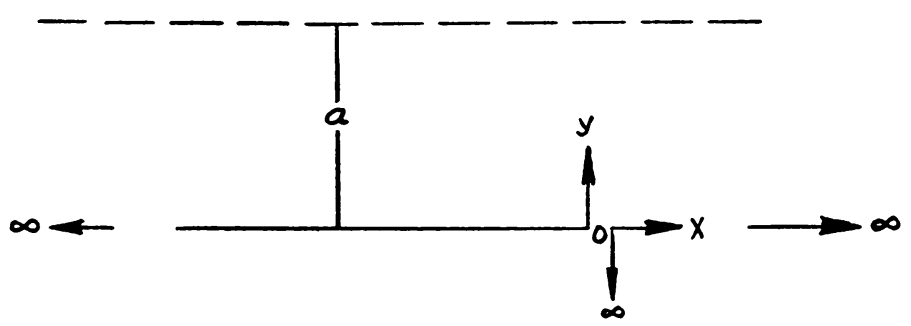

FIG. 1.

from the begimning. This gives us as fundamental equations of motion

$$
\begin{aligned}
& \phi_{x r}+\phi_{\nu \nu}-k^{2} \phi=0 \text { in the fluid medium, } \\
& \phi_{n}=0 \quad \text { on a rigid surface, and } \\
& \phi_{\nu}=\beta \phi \quad \beta=\omega^{2} \text {,! } \quad \text { on a free surface. }
\end{aligned}
$$

Equation (2.1) possesses the following types of bounded solutions. For $x \rightarrow \infty, y<a$

$$
\phi=\exp \left[i k z+\beta y \pm i\left\{\beta^{2}-k^{2}\right\}^{1 / 2} x\right]
$$

while for $x \rightarrow-\infty, 0 \leq y \leq a$

$$
\exp \left(i k z \pm i_{\kappa} x\right) \cosh \alpha_{0} y / a
$$

where

$$
\kappa=\left\{\left(\alpha_{0} / a\right)^{2}-k^{2}\right\}^{1 / 2}
$$

and

$$
\alpha \sinh \alpha=\beta a \cosh \alpha
$$

has the smallest real roots $\pm \alpha_{0}$. Eq. (2.2) also has an infinite sequence of imaginary roots $i \alpha_{n}, \alpha_{-n}=-\alpha_{n}, n=1,2, \cdots$. Wave motion exists if $\beta>k$ and $\left|\alpha_{0} / a\right|>k$.

III. Derivation of the Integral Equation. Following methods which have been discussed elsewhere (1), (2), (3), it is a fairly straightforward task to formulate the integral equation which describes the boundary value problem considered in Sec. II. In view of the fact that we have suppressed the $z$ dependence in $\phi$, we have essentially a two dimensional problem. We shall divide the half space $y \leq a$ into two regions: (a) the strip $0 \leq$ $y \leq a,-\infty<x<\infty$ which we shall henceforth refer to as $R_{1}$ and $(b)$ the half plane $y \leq 0,-\infty<x<\infty$ which we shall refer to as $R_{2}$. Since we require the use of the Helmholtz theorem in its improper form to provide representations of $\phi(x, y)$ in $R_{1}$ and $R_{2}$ in terms of $\phi_{\nu}(x, 0)$, some statements regarding the growth of $\phi$ as $r \rightarrow \infty$ will be in order $\left(r=\left[x^{2}+y^{2}\right]^{1 / 2}\right)$. To begin with, in $R_{1}$ for $x \rightarrow \infty, \phi(x, y)$ is asymptotic to

$$
\left[\gamma_{1} \exp \left(i_{\kappa} x\right)+\gamma_{2} \exp \left(-i_{\kappa} x\right)\right] \cosh \alpha_{n} y_{1}^{\prime} a .
$$

This is tantamount to the statement that the asymptotic form of $\phi$ for $x \rightarrow-\infty$ is the bounded solution which would have been obtained had the barrier extended to infinity on the right. For $x \rightarrow \infty$ and $y \leq a$, we specify the asymptotic form of $\phi$ as

$$
\left[\gamma_{3} \exp (i \sigma x)+\gamma_{4} \exp (-i \sigma x)\right] \exp (\beta y)
$$


where $\sigma=\left\{\beta^{2}-k^{2}\right\}^{1 / 2}$ and the positive root is to be understood. This again is a statement that for a channel of infinite depth without a barrier (3.2) is the bounded solution. One final task remains and that is to specify the nature of $\phi$ as $r \rightarrow \infty, x<0$ and $y<0$. In this we are not as fortunate as we were in the other two cases. We shall assume (and verify with the solution of the problem) that $\phi=0\left[\exp \left(k_{1} r\right)\right]$ for $y<0, x<0, r \rightarrow \infty$ where $k_{1}<k$. This condition will enable us to write a representation for $\phi$ in $R_{2}$, that is for $y \leq 0$. One final assumption is required and that is the statement that $\phi$ and $\phi_{\nu}$ are integrable for all finite $x$ - again subject to final verification.

If we introduce a Green's function $G^{(1)}\left(x, y, x^{\prime}, y^{\prime}\right)$ associated with Eq. (2.1) and which satisfies the boundary conditions

and

$$
G_{y}^{(1)}=\beta G^{(1)} \quad y=a, \quad-\infty<x<\infty
$$

$$
G_{v}^{(1)}=0 \quad y=0, \quad-\infty<x<\infty
$$

and the further conditions at infinity that $G^{(1)} \rightarrow 0$ for $x^{\prime}-x \rightarrow \infty$ while for $x-x^{\prime} \rightarrow \infty$

$$
G^{(1)}=\frac{4 \alpha_{0} \cosh \alpha_{0} y / a \cosh \alpha_{0} y^{\prime} / a \sin \kappa\left(x-x^{\prime}\right)}{a \kappa\left(2 \alpha_{0}+\sinh 2 \alpha_{0}\right)}
$$

we have as a representation for $\phi$ in $R_{1}$

$\phi(x, y)=\left[\cosh \alpha_{0} y / a\right]\left[\gamma_{1} \exp (i \kappa x)+\gamma_{2} \exp (-i \kappa x)\right]$

Here (3)

$$
-\int_{0}^{\infty} G^{(1)}\left(x, y, x^{\prime}, 0\right) \phi_{y^{\prime}}\left(x^{\prime}, 0\right) d x^{\prime} .
$$

$$
\begin{aligned}
G^{(1)}\left(x, y, x^{\prime}, y^{\prime}\right)=\sum_{n=1}^{\infty} & \frac{2 \alpha_{n}\left(\cos \alpha_{n} y / a\right)\left(\cos \alpha_{n} y^{\prime} / a\right) \exp \left[-\left|x-x^{\prime}\right|\left\{k^{2}+\alpha_{n}^{2} / a^{2}\right\}^{1 / 2}\right]}{a\left(2 \alpha_{n}+\sin 2 \alpha_{n}\right)\left\{\alpha_{n}^{2} / a^{2}+k^{2}\right\}^{1 / 2}} \\
& +0 \quad \text { if } \quad x^{\prime}>x \text { or } \\
& +\frac{4 \alpha_{0}\left(\cosh \alpha_{0} y / a\right)\left(\cosh \alpha_{0} y^{\prime} / a\right) \sin \kappa\left(x^{\prime}-x\right)}{a \kappa\left(2 \alpha_{0}+\sinh 2 \alpha_{0}\right)} \text { if } \quad x^{\prime}<x .
\end{aligned}
$$

For the region $R_{2}$ we have in a similar fashion

$$
\phi(x, y)=\int_{0}^{\infty} G^{(2)}\left(x, y, x^{\prime}, 0\right) \phi_{y^{\prime}}\left(x^{\prime}, 0\right) d x^{\prime}
$$

where

$$
\begin{aligned}
G^{(2)}\left(x, y, x^{\prime}, y^{\prime}\right)=\frac{1}{2 \pi}\left[K_{0}\left(k\left\{\left(x-x^{\prime}\right)^{2}+\left(y-y^{\prime}\right)^{2}\right\}^{1 / 2}\right)\right. & \\
& \left.+K_{0}\left(k\left\{\left(x-x^{\prime}\right)^{2}+\left(y+y^{\prime}\right)^{2}\right\}^{1 / 2}\right)\right] .
\end{aligned}
$$

The function $K_{0}(\delta)$ is the solution of the differential equation

$$
y_{\delta \delta}+y_{\delta} / \delta-y=0
$$

which is $0(\log \delta), \delta \rightarrow 0^{+}$. It is a Bessel function of the second kind which possesses the property that it is of the order $\exp (-\delta) / \delta^{1 / 2}$ for $\delta \rightarrow \infty$. This last order condition accounts for the choice of the constant $k_{1}$. On the basis of these two representations of 
$\phi(x, y)$ in $R_{1}$ and $R_{2}$ we can form an integral equation of the Wiener-Hopf type. We note that $\phi(x, y)$ is continuous at $y=0$ for all $x>0$. We have then for $x>0$

$\gamma_{1} \exp \left(i_{\kappa} x\right)+\gamma_{2} \exp \left(-i_{\kappa} x\right)$

$$
-\int_{0}^{\infty}\left[G^{(1)}\left(x, 0, x^{\prime}, 0\right)+G^{(2)}\left(x, 0, x^{\prime}, 0\right)\right] \phi_{\nu},\left(x^{\prime}, 0\right) d x^{\prime}=0 .
$$

IV. The Fourier Transform of the Integral Equation. We now define Eq. (3.6) to read for all $x$

$$
I(x)=J(x)-\int_{-\infty}^{\infty}\left[G^{(1)}\left(x, 0, x^{\prime}, 0\right)+G^{(2)}\left(x, 0, x^{\prime}, 0\right)\right] L\left(x^{\prime}\right) d x^{\prime}
$$

Here

$$
\begin{array}{rlrl}
I(x) & \equiv 0 \quad \text { for } \quad x>0 & \\
J(x) & \equiv \gamma_{1} \exp \left(i_{\kappa} x\right)+\gamma_{2} \exp \left(-i_{\kappa} x\right) & & x>0 \\
& \equiv 0 & & x<0 \\
L(x) & \equiv 0 & & x<0 \\
& \equiv \phi_{\nu^{\prime}}(x) & & x>0
\end{array}
$$

Clearly then $I(x)=0[\exp (k x)]$ for $x \rightarrow-\infty$ and $L(x)=0\left[\exp k_{1} x\right]$ for $x \rightarrow \infty$. Actually $L(x)$ is bounded for $x \rightarrow \infty$, being of the form $\exp ( \pm i \sigma x)$ but this last description of $\phi(x, 0)$ for $x \rightarrow \infty$ need not be stated explicitly.

Let us now introduce the Fourier transforms of $I(x), J(x)$ and $L(x)$ as well as those of $G^{(1)}(x, 0,0,0)$ and $G^{(2)}(x, 0,0,0)$. Henceforth we shall use the notation $I(w)$ to denote the Fourier transform of $I(x), J(w)$ for $J(x)$, etc. We have from Eq. (4.1)

$$
I(w)=\frac{\gamma_{1}}{i(w-\kappa)}+\frac{\gamma_{2}}{i(w+\kappa)}-L(w)\left[G^{(1)}(w)+G^{(2)}(w)\right]
$$

where

$$
\begin{aligned}
G^{(1)}(w) & =\int_{-\infty}^{\infty} \exp (-i w x) G^{(1)}\left(x, y, 0, \dot{y}^{\prime}\right) d x \\
& =\frac{\cosh \rho \sin \sinh \rho(t-a)+\rho \cosh \rho(t-a)]}{\rho[\rho \sinh \rho a-\beta \cosh \rho a]}
\end{aligned}
$$

and $(s, t)=\left(y, y^{\prime}\right)$ if $y<y^{\prime}$ or $\left(y^{\prime}, y\right)$ if $y>y^{\prime}$. Furthermore

$$
\begin{aligned}
G^{(2)}(w) & =\int_{-\infty}^{\infty} \exp (-i w x) G^{(2)}\left(x, y, 0, y^{\prime}\right) d x \\
& =\left\{\exp \left[-\rho\left|y-y^{\prime}\right|\right]+\exp \left[-\rho\left|y+y^{\prime}\right|\right]\right\} / 2 \rho .
\end{aligned}
$$

where $\rho=\left\{k^{2}+w^{2}\right\}^{1 / 2}$. The branch of $\rho$ has been chosen which reduces to $k$ when $w$ $=0$.

We now examine the regions of regularity of the various transforms. First $I(w)$ is 
regular in the upper half plane $v>-k(w=u+i v), J(w)$ is regular in the lower half plane $v<0$, and $L(w)$ in the lower half plane $v<-k_{1}$. This follows from the assumed integrability for finite $x$ of $I(x), L(x)$ and $J(x)$ as well as their growths for $|x| \rightarrow \infty$. Similarly $G^{(1)}(w)$ is regular in the strip - $\left\{k^{2}+\alpha_{1}^{2} / a^{2}\right\}^{1 / 2}<v<0$ while $G^{(2)}(w)$ is regular in the strip $-k<v<k$. There is then a common strip of analyticity for all of these transforms, the application of the Fourier transform to Eq. (3.4) is permissible and we obtain Eq. (4.2). ("pon simplifying we get

$$
I(w)=\frac{\gamma_{1}}{i(w-\kappa)}+\frac{\gamma_{2}}{i(w+\kappa)}-K(w) L(w)
$$

where

$$
K(w)=[1-\beta / \rho][\exp a \rho]^{i}[\rho \sinh \rho a-\beta \cosh \rho a] .
$$

Following Wiener and IIopf (5), Eq. (4.3) may be rewritten

$$
\begin{aligned}
K_{+}(w) I(w)-\gamma_{1} \frac{K_{+}(w)-K(\kappa)}{i(w-\kappa)}-\gamma_{2} & \frac{K_{+}(w)-K(-\kappa)}{i(w+\kappa)} \\
& =\frac{\gamma_{1} K_{+}(\kappa)}{i(w-\kappa)}+\frac{\gamma_{2} K(-\kappa)}{i(w+\kappa)}-L(w) K_{-}(w)
\end{aligned}
$$

where $K_{+}(w)$ is regular in the upper half plane $v>-k$ while $K_{-}(w)$ is regular in the lower half plane $v<0$. Eq. (4.4), as a result of an argument of analytic continuation described by Wiener and Hopf, implies that each side if individually an entire function. Our next goal is to determine the explicit forms of $K_{-}(w)$ and $K_{+}(w)$ as well as the entire function of separation.

V. The Determination of $K_{-}(w)$ and $K_{+}(w)$. Thus far we have pursued a familiar course to arrive at Eq. (4.4). From this point on we encounter a number of unfamiliar situations. In the decomposition of $K(u)$ we have three individual components to consider:

$$
\begin{aligned}
& \text { (a) } \rho \sinh \rho a-\beta \cosh \rho a \\
& \text { (b) } \exp (\rho a) \\
& \text { (c) } 1-\beta / \rho
\end{aligned}
$$

Component (a) is familiar and has been discussed in (2). The type of factoring we employ implies that the portion of (a) which is regular in some lower half plane also has a regular reciprocal in the same half plane. The same remark may be addressed to the upper half plane factor. We have then

$$
\rho \sinh \rho a-\beta \cosh \rho a=M_{+}(w) / M_{-}(w)
$$

whera $M_{+}(w)$ is regular in the upper half plane $v>-\alpha_{1}\left\{1+a^{2} k^{2} / \alpha_{1}^{2}\right\}^{1 / 2}$ and $M_{-}(w)$ is regular in the lower half plane $v<0$. Here

and

$$
M_{+}(w)=\prod_{m=1}^{\infty}\left[\left\{1+a^{2} l_{i}^{2} / \alpha_{m}^{2}\right\}^{1 / 2}-i a w / \alpha_{m}\right] \exp (i a w / m \pi)
$$

$$
1 / M_{-}(w)=\prod_{m=1}^{\infty}\left[\left\{1+a^{2} k^{2} / \alpha_{m}^{2}\right\}^{1 / 2}+i a w / \alpha_{m}\right] \exp (-i a w / m \pi) \frac{a^{2} \beta}{\alpha_{0}^{2}}\left(w^{2}-\kappa^{2}\right) .
$$


For

$$
\begin{gathered}
|w| \rightarrow \infty \quad I m w>-\left\{\alpha_{1}^{2}+a^{2} k^{2}\right\}^{1 / 2} \\
M_{+}(w)=0[w \Gamma(-i a w / \pi) \exp (-\gamma i a w / \pi)]^{-1}
\end{gathered}
$$

where $\gamma$ is the Euler-Mascheroni constant, while for $|w| \rightarrow \infty, \operatorname{Im} w<0$

$$
M_{-}(w)=0\left[\exp (+i a w \gamma / \pi) \Gamma(i a w / \pi) w^{-1}\right] .
$$

Now we turn to (b). The decomposition of $\exp (\rho a)$ multiplicatively is equivalent to the decomposition of additively. $\rho=\left\{w^{2}+k^{2}\right\}^{1 / 2}$ is regular in the entire $w$ plane provided cuts are introduced along the imaginary axis from $k$ to infinity and $-k$ to negative infinity. We define

$$
\left\{w^{2}+k^{2}\right\}^{1 / 2}=\left|w^{2}+k^{2}\right|^{1 / 2}[\exp \{i / 2 \arg (w-i k)+i / 2 \arg (w+i k)\}]
$$

where

$$
\begin{gathered}
-3 \pi / 2<\arg (w-i k)<\pi / 2 \\
-\pi / 2<\arg (w+i k)<3 \pi / 2
\end{gathered}
$$

With these definitions at hand we turn to the decomposition of $1 / \rho$ from which we can readily obtain that of $\rho$. From Cauchy's theorem we have

$$
2 \pi i /\left(w^{2}+k^{2}\right)^{1 / 2} \equiv \int_{C} d z_{\prime}^{\prime}(z-w)\left(z^{2}+k^{2}\right)^{1 / 2}
$$

where $C$ is a closed rectangular path in the strip $-k<\operatorname{Im} z<k$ whose vertical contributions disappear as they recede to infinity. The upper side of the rectangle may be deformed into a path along the branch cut from $k$ to infinity, while the lower one goes into a path over the lower cut and we get

$$
\pi i /\left(w^{2}+k^{2}\right)^{1 / 2}=\int_{k}^{\infty} d y /(w-i y)\left(y^{2}-k^{2}\right)^{1 / 2}-\int_{k}^{\infty} d y /(w+i y)\left(y^{2}-k^{2}\right)^{1 / 2}
$$

This provides a separation of the required type-the first term being regular in the lower half plane $v<k$, the second in the upper half plane $v>-k$. For our purposes, however, we require more explicit information, particularly as to the nature of these integrals as $|w| \rightarrow \infty$ and we therefore proceed with the evaluation of these integrals.

As a matter of notation we write

$$
\pi i\left(w^{2}+k^{2}\right)^{-1 / 2}=f(w)+f(-w)
$$

where

$$
f(w)=\int_{k}^{\infty} d y /(w-i y)\left(y^{2}-k^{2}\right)^{1 / 2}=\left(w^{2}+k^{2}\right)^{-1 / 2} \log [(1+t) /(1-t)]
$$

and

$$
t=(w+i k)^{1 / 2}(w-i k)^{-1 / 2} .
$$

Upon using the definitions of the $\arg (w+i k)$ and $\arg (u-i k)$ which we wrote above, 
we find that the cut $w$ plane (Figure 2) maps into the upper half of the $t$ plane by means of (5.3). Further the transformation $z=(1+t) /(1-t)$ is bilinear and hence maps the upper half plane $I m t>0$ into the upper half plane $\operatorname{Imz}>0$ (Figure 2). We therefore define the branch of the logarithm by $0 \leq \arg z \leq \pi$. Given this information, we may verify that $f(w)$ is regular in the lower half plane $v<k$. The factor $\left(w^{2}+k^{2}\right)^{-1 / 2}$ multiplying the logarithm in $f(w)$ changes sign as we cross the lower branch cut. But the

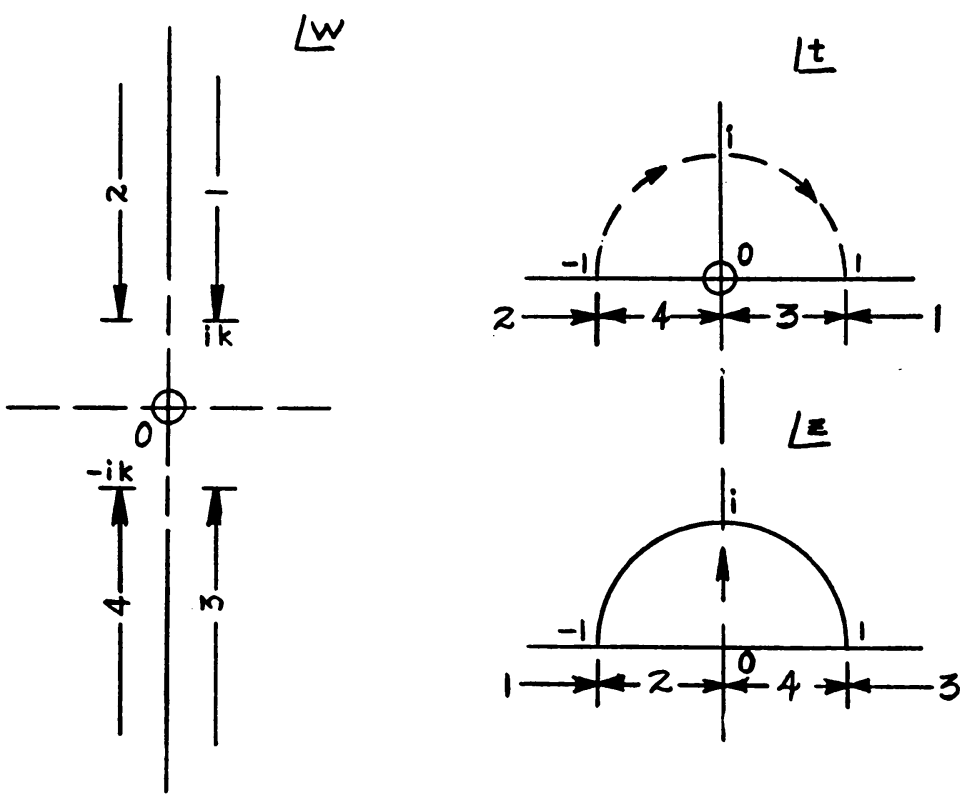

FIG. 2.

$\arg z=0$ on this cut so that the real part of $f(w)$ is continuous. In crossing the branch cut $\log |z|$ becomes $-\log |z|$ so that the imaginary part of $f(w)$ is continuous. Hence $f(w)$ is regular in the required lower half plane. On the upper branch out the $\arg z=\pi$ and hence the sign of the radical multiplier introduces a discontinuity.

Similar comments apply to the function $f(-w)$. The transformation $t^{\prime}=(w-i k)^{1 / 2}$. $(w+i k)^{-1 / 2}$ sends the cut plane into the half plane Imt $<0$ and the transformation $z^{\prime}=\left(1-t^{\prime}\right)\left(1+t^{\prime}\right)^{-1}$ takes the half plane Imt $<0$ into the upper half plane $I m z^{\prime}>0$ with the upper branch cut mapped into the positive $z^{\prime}$ axis. We put $0 \leq \arg z^{\prime} \leq \pi$ to define the branch of the logarithm in $f(-w)$, that is

$$
f(-w)=\left(w^{2}+k^{2}\right)^{-1 / 2} \log (1-t)(1+t)^{-1}
$$

and we find that $f(-w)$ is now regular in the upper half plane $v>-k$.

As a check, we have in the strip $-k<v<k$

$$
\begin{aligned}
f(w)+f(-w) & =\left(w^{2}+k^{2}\right)^{-1 / 2}\left[\log z+\log z^{\prime}\right] \\
& =\left(w^{2}+k^{2}\right)^{-1 / 2}\left[\log \left|z z^{\prime}\right|+i \arg z+i \arg z^{\prime}\right] .
\end{aligned}
$$


Since $z=-1 / z^{\prime}$, this last expression reduces to the one with which we started. Hence if we write $\exp \left[a\left(w^{2}+k^{2}\right)^{1 / 2}\right]$ in product form as we have already described, we find that

where

$$
\exp \left[a\left(u^{2}+k^{2}\right)^{1 / 2}\right]=N_{-} / N_{+}
$$

$$
\begin{aligned}
N_{-}(w) & =\exp \left[a\left(w^{2}+k^{2}\right) f(w) / \pi i\right] \\
1 / N_{+}(w) & =\exp \left[a\left(w^{2}+k^{2}\right) f(-w) / \pi i\right] .
\end{aligned}
$$

The asymptotic forms of $f(w)$ and $f(-w)$ will be required at a later point in our work and since the analytical machinery is now developed, we shall provide these forms. Note first that

$$
\log (1+t)(1-t)^{-1}=\log \left\{i\left[w+\left(w^{2}+k^{2}\right)^{1 / 2}\right] / k\right\} .
$$

Further for $u>0, v<-k,-\pi / 2<\arg \left(w^{2}+k^{2}\right)^{1 / 2}<0$ and $-\pi / 2<\arg u<0$. Hence for $|w| \rightarrow \infty, v<-k, u>0$,

$$
\log \left\{i\left[w+\left(w^{2}+k^{2}\right)^{1 / 2}\right] / k\right\}=\log [(2 i w / k)+(i k / 2 w)]
$$

up to terms of $0(1 / w)$. This in turn has the expansion

$$
(\log 2 i w / k)-k^{2} / 4 w^{2} \text {. }
$$

Now the choice of the definition of the logarithm is dictated by the fact that the argument of the logarithm in (5.4) lies between the limits 0 and $\pi$. Hence if we consider the principal branch of the logarithm of $w$ we see that $\log i=i \pi / 2$ and hence

$$
\log (1+t)(1-t)^{-1}=i \pi / 2+\log 2 / k+\log w+0\left(1 / w^{2}\right)
$$

in this quadrant. If we now turn to the third quadrant, we see that we get certain minor changes but the same dominant term. In this quadrant $0<\arg \left(w^{2}+k^{2}\right)^{1 / 2}<\pi / 2$ while $-\pi<\arg w<-\pi / 2$. Then

$$
\begin{aligned}
\log (1+t)(1-t)^{-1} & =\log \left[i\left\{w+\left(w^{2}+k^{2}\right)^{1 / 2}\right\} / k\right] \\
& =-\log 2 i w / k+0\left(1 / w^{2}\right)
\end{aligned}
$$

for $u<0, v<-k,|w| \rightarrow \infty$. But again the logarithmic term has an argument between 0 and $\pi$ and since the arg $w$ lies between $-\pi$ and $-\pi / 2$, the negative of this argument lies between $\pi / 2$ and $\pi$. Hence as we might have surmised

$$
\log (1+t)(1-t)^{-1}=-i \pi / 2-\log w-\log 2 / k+0\left(1 / w^{2}\right)
$$

for $|w| \rightarrow \infty, v<+k, u<0$. This change in the asymptotic form of the logarithm of $\log (1+t)(1-t)^{-1}$ in the third quadrant does not change the dominant term in the asymptotic form of $f(w)$ however and $\left(5.4^{\prime}\right)$ is still in force. Similarly

$$
f(-w)=-\left[\log w+\log 2 / k-i \pi / 2+0\left(1 / w^{2}\right)\right] / w
$$

for $|w| \rightarrow \infty, v>-k$. From this we conclude that

$$
N_{-}(w)=\exp [a w / 2+(a w / \pi i)(\log 2 / k)+(a w / \pi i) \log w]
$$

if we retain the non-vanishing terms for $|w| \rightarrow \infty, v<+k$, while

$$
1 / N_{+}(w)=\exp [(-a w / 2)-(a w / \pi i)(\log 2 / k)-(a w / \pi i)(\log w)]
$$

for $|w| \rightarrow \infty, v>-k$. 
The factoring of $1-\beta /\left(w^{2}+k^{2}\right)^{1 / 2}$ into the product of two terms, one of which is regular in the lower half plane $v<0$, while the other of which is regular in the upper half plane $v>-k$ is guaranteed by the original work of Wiener and Hopf. However, rather than apply their original methods, we shall follow an approach which will enable us to express the factors in terms of $f(w)$ and $f(-w)$. Again following the lead from the form $(b)$, we write

$$
1-\beta /\left(w^{2}+k^{2}\right)^{1 / 2}=\exp \left[\ln \left\{1-\beta /\left(w^{2}+k^{2}\right)^{1 / 2}\right\}\right]
$$

where we choose the branch of $\left(w^{2}+k^{2}\right)^{1 / 2}$ which reduces to $k$ for $w=0$ as well as the principal branch of the logarithm. Instead of decomposing the logarithmic factor additively, we shall decompose its derivative in this fashion since differentiation will not alter the open strip of regularity. Hence we shall examine first

$$
\begin{aligned}
& \frac{d}{d w} \ln \left[1-\beta\left(w^{2}+k^{2}\right)^{1 / 2}\right]=w \beta /\left(w^{2}+k^{2}\right)\left[\left\{w^{2}+k^{2}\right\}^{1 / 2}-\beta\right] \\
& \left.=-\frac{1}{2(w-i k)}-\frac{1}{2(w+i k)}+\frac{w}{w^{2}-\sigma^{2}}-\frac{w}{\beta\left\{w^{2}\right.}+k^{2}\right\}^{1 / 2}+\frac{w\left\{w^{2}+k^{2}\right\}^{1 / 2}}{\beta\left\{w^{2}-\sigma^{2}\right\}} .
\end{aligned}
$$

Now of these five terms, we can state the following. The first and third are regular in the lower half plane $v<0$, while the second is regular in the upper half plane $v>-k$. The fourth and fifth terms are regular only in the strips $-k<v<k$ and $-k<v<0$ respectively. The fourth term however is the derivative of $-\left(w^{2}+k^{2}\right)^{1 / 2} / \beta$ which has been discussed in (b). The fifth term may be decomposed as follows:

$$
\frac{w\left\{w^{2}+k^{2}\right\}^{1 / 2}}{\beta\left\{w^{2}-\sigma^{2}\right\}}=\frac{\left\{w^{2}+k^{2}\right\}^{1 / 2}}{2 \beta}\left\{\frac{1}{w+\sigma}+\frac{1}{w-\sigma}\right\} .
$$

We know how to decompose $\left(w^{2}+k^{2}\right)^{1 / 2}$ additively so that this last term breaks up into four terms, two of which are regular in the lower half plane $v<0$, while the other two are still regular in the strip $-k<v<0$. But the simple poles in these last terms may be extracted quite readily so that we finally have that the portion of (5.5) which is regular in the appropriate lower half plane is

$$
\begin{aligned}
U_{-}(w)= & -\frac{1}{2(w-i k)}+\frac{w}{w^{2}-\sigma^{2}}-\frac{1}{\pi \beta i} \frac{d}{d w}\left(w^{2}+k^{2}\right) f(w) \\
& +\frac{\left(w^{2}+k^{2}\right) f(w)}{2 \beta \pi i(w+\sigma)}+\frac{\left(w^{2}+k^{2}\right) f(w)}{2 \beta \pi i(w-\sigma)} \\
& +\frac{\beta f(+\sigma)}{2 \pi i(w+\sigma)}+\frac{\beta f(-\sigma)}{2 \pi i(w-\sigma)} .
\end{aligned}
$$

On the other hand, the term

$$
\begin{aligned}
U_{+}(w)= & -\frac{1}{2(w+i k)}-\frac{1}{\pi \beta i} \frac{d}{d w}\left(w^{2}+k^{2}\right) f(w) \\
& +\frac{1}{2 \beta \pi i(w+\sigma)}\left[\left(w^{2}+k^{2}\right) f(-w)-\beta^{2} f(+\sigma)\right] \\
& +\frac{1}{2 \beta \pi i(w-\sigma)}\left[\left(w^{2}+k^{2}\right) f(-w)-\beta^{2} f(-\sigma)\right]
\end{aligned}
$$


is regular in the appropriate upper half plane. Hence

$$
1-\beta / \rho=\left[\exp \int^{w} U_{-}(w) d w\right]\left[\exp \int^{10} U_{+}(w) d w\right]
$$

where the first factor is regular in the appropriate lower half plane while the second is regular in the appropriate upper half plane. The constants of integration are chosen so that the left side matches the right side in the strip of regularity.

The asymptotic forms of exp $\int^{\prime \prime} C_{-}^{*}(w) d w$ and $\exp \int^{w} L_{+}(w) d w$ are readily determined from our results in $(b)$. We have for $|w| \rightarrow \infty, v<0$

while

$$
L_{-}(u)=1 / w+0\left(1 / w^{2}\right)
$$

for $|w| \rightarrow \infty, v>-k$. IIence

$$
l^{\circ}(w)=-1 / w+0\left(1 / w^{2}\right)
$$

$$
\int^{w} \exp L_{-}-(u) d w=c w
$$

and

$$
\int^{w} \exp l_{*}^{*}(x) d w=c_{1} / w
$$

in these respective cases where the constants $c_{1}$ and $c$ satisfy the relation $c c_{1}=1$. The precise values of the constants do not concern us here.

Now we may put together the factors $(a),(b)$ and $(c)$ to determine $K_{-}(w)$. In order to obtain an entire function of separation which is of algebraic growth, we introduce into $K_{-}(w)$ a factor exp $\chi(u)$ where $\chi(w)$ is to be chosen to make $K_{-}(w)$ of algebraic order for $v<k_{1},|w| \rightarrow \infty$. This process has already been described elsewhere (1). We have then, for $v<k_{1},|w| \rightarrow \infty$

$$
K_{-}(w)=0\left(w^{-1,2}\right) \operatorname{cxp}[(i a w ; \pi)(\gamma-1+\ln a k / 2 \pi)+\chi(w)] .
$$

From this we see that if $\chi(w)$ is chosen as

$$
\chi(w)=[1-\gamma-\ln (a k / 2 \pi)](i a w / \pi)
$$

$K_{-}(w)=0\left(w^{-1 / 2}\right)$ in this lower half plane. Upon examining $K_{+}(w)$ for $|w| \rightarrow \infty, v>-k$ we find that $K_{+}(w)=0\left(w^{+1 / 2}\right)$. Hence the factoring of $K(w)$ and the asymptotic forms of the fuctors are known explicitly.

In order to determine $E(w)$, the entire function of separation, we merely apply an extended form of Liouville's theorem. For example, for $|w| \rightarrow \infty, v<0, E(w)=$ $0\left(w^{-1}\right)$, while for $|w| \rightarrow \infty, v>-k, E(w)=0\left(w^{1 / 2}\right)$ as we can readily see from Eq. (4.4). From this we see that since $E(w)$ is a polynomial, $E(w)$ is identically zero.

Earlier in our work we had assumed that $\phi_{\nu}(x, 0)$ was integrable from $x=0$ to any finite $x$. In view of the fact that $I(w)=0\left(w^{-1}\right)$ and $J(w)=0\left(w^{-1 / 2}\right)$ for $|w| \rightarrow \infty, v$ suitable limited, we see that $I(x)$ is bounded for $x \rightarrow 0^{-}$, while $\phi_{\nu}(x, 0)=0\left(x^{-1 / 2}\right)$ for $x \rightarrow 0^{+}$, thus verifying the integrability for finite $x$ for these functions. The precise forms of $\phi$ will be investigated in the next section.

VI. The Determination of $\phi(x, y)$. In order to determine $\phi(x, y)$ we return to the Helmholtz representations for $\phi$ in $R_{1}$ and $R_{2}$. For $R_{1}$ we have from (3.5)

$\phi(x, y)=\left[\cosh \alpha_{n} y / a\right]\left[\gamma_{1} \exp (i \kappa . x)+\gamma_{2} \exp (-i \kappa x)\right]$

$$
-\frac{1}{2 \pi} \int_{c} G^{(1)}(w, y, 0) L(w) \exp (i u x) d w .
$$


Here $G^{(1)}(w, y, 0)$ is the bilateral Fourier transform of $G^{(1)}(x, y, 0,0)$ an analytic function of $w$ in the strip $-\left\{k^{2}+\alpha_{0}^{2} / a^{2}\right\}^{1 / 2}<v<0$. The convolution is justified here since $L(w)$ is the transform of $L(x) \exp (v x)$ which is $L(-\infty, \infty)$ for $-k<v<0$ and $0<y$ $<a(6)$. The path $C$ is drawn in the strip of analyticity $-k<v<0$ and the evaluation of the integral depends on whether $x \lessgtr 0$. If $x<0$, we close below by a sequence of semicircular arcs passing between the poles of $G^{(1)} .(u, y, 0)$ on the negative imaginary axis. In this case the integral becomes

$$
\begin{aligned}
-\frac{1}{2 \pi} \int_{c}\left[\frac{\gamma_{1} K_{+}(\kappa)}{i(w-\kappa)}+\right. & \left.+\frac{\gamma_{2} K_{+}(-\kappa)}{i(w+\kappa)}\right]\left[\frac{\left.\rho \cosh \rho(y-a)+\beta \sinh \frac{\rho(y-a)}{K_{-}(w)[\rho \sinh \rho a-\beta}\right] \frac{\exp (i w x) d u}{\rho}}{\cosh \rho a]}\right. \\
& =\sum_{n=1}^{\infty}\left[\frac{\gamma_{1} K_{+}(\kappa)}{\left(-i w_{n}-\kappa\right)}+\frac{\gamma_{2} K_{-}(-\kappa)}{\left(\kappa-i w_{n}\right)}\right] \frac{\exp \left(w_{n} x\right)\left(\rho_{n}^{2}+a^{2} \beta^{2}\right) \exp \left(w_{n} x\right)}{i K_{-}\left(-i w_{n}\right) a w_{n}\left(a \beta-a^{2} \beta^{2}-\alpha_{n}^{2}\right)}
\end{aligned}
$$

where $w_{n}=\left\{\alpha_{n}^{2} / a^{2}+k^{2}\right\}^{1 / 2}$. The closing of the path $C$ in the manner described above is permitted since the integrand is $0\left[w^{-3 / 2} \exp (-v x)\right]$ for $|w| \rightarrow \infty, v<0$.

For $x>0$, the path $C$ in $\mathrm{Eq} .(6.1)$ is closed above. The singularities in the upper half plane are a branch point at $w=i k$ and four poles at $w= \pm \sigma$ and $\pm \pi$. We introduce the positive imaginary axis from $i k$ to $i \infty$ as a branch cut. The phase of $\rho$ has already been discussed on this cut, so the closing of $C$ implies quarter circles on each side of the branch cut as well as integrals along the branch cut. This then supplies us with a path along which the integrand is single valued and analytic and Cauchy's theorem may be applied for the evaluation of the integral. When we do so we get the residues at the poles $\pm \sigma$ and $\pm \kappa$ and an integral along the branch cut. To do this we replace

and the integral along $C$ becomes

$$
K_{-}(w)=\frac{\exp (a \rho)(1-\beta / \rho) K_{+}(w)}{\rho \sinh \rho a-\beta \cosh \rho a}
$$

$$
-\frac{1}{2 \pi} \int_{c}\left[\frac{\gamma_{1} K_{+}(\kappa)}{i(w-\kappa)}+\frac{\gamma_{2} K_{+}(-\kappa)}{i(w+\kappa)}\right]\left[\frac{\rho \cosh \rho(y-a)+\beta \sinh \rho(y-a)}{(\exp a \rho)(\rho-\beta) K_{+}(w)}\right] \exp (i w x) d w .
$$

The contributions from the quarter circles drop out as their radii becomes infinite and we are left with an integral along the cut as well as four residues. On the right side of the cut $\arg =\pi / 2$ while on the left side of the cut $\arg \rho=-\pi / 2$. Hence

$$
\int_{c}+\int_{b r}=\sum \text { residues }
$$

or

$$
\begin{aligned}
\int_{c}=-\left[\cosh \alpha_{0} y / a\right]\left[\gamma_{1} \exp (i \kappa x)+\gamma_{2} \exp (-i \kappa x)\right] \\
-\left[\frac{\gamma_{1} K_{+}(\kappa)}{\sigma-\kappa}+\frac{\gamma_{2} K_{+}(-\kappa)}{\sigma+\kappa}\right] \frac{\beta^{2} \exp \frac{\beta(y-2 a) \exp (i \sigma x)}{\sigma K_{+}(\sigma)}}{+} \\
+\left[\frac{\gamma_{1} K_{+}(\kappa)}{-(\sigma+\kappa)}+\frac{\gamma_{2} K_{+}(-\kappa)}{\kappa-\sigma}\right] \frac{\beta^{2} \exp \beta(y-2 a) \exp (-i x)}{\sigma K_{+}(\sigma)} \\
-\frac{i}{\pi} \int_{k}^{\infty}\left[\frac{\gamma_{1} K_{+}(\kappa)}{(i v-\kappa)}+\frac{\gamma_{2} K_{+}(-\kappa)}{(i v+\kappa)}\right]\left[\frac{T \cos T(y-a)+\beta \sin T(y-a)}{K_{+}(i v)\left(v^{2}+\sigma^{2}\right)}\right] \\
+\left[\frac{\beta \cos T a+T \sin T a}{\exp (v x)}\right] d v
\end{aligned}
$$

where $T=\left(v^{2}-k^{2}\right)^{1 / 2}$. 
Now we examine $\phi(x, y)$ in $R_{2}$. Here we use the representation

$$
\begin{aligned}
\phi(x, y) & =-\int_{0}^{\infty} G^{(2)}\left(x, y, x^{\prime}, 0\right) \phi_{y^{\prime}}\left(x^{\prime}, 0\right) d x \\
& =-\frac{1}{2 \pi} \int_{c} \frac{\exp (i w x) \exp (\rho y) L(w) d w}{\rho} \\
& \left.=-\frac{1}{2 \pi} \int_{c} \frac{d w}{\rho K_{-}(w)} \frac{\exp (i w x+\rho y)}{(w-\kappa)}+\frac{\gamma_{1} K(\kappa)}{(w+\kappa)}\right]
\end{aligned}
$$

and again we distinguish the two cases, that is $x \gtrless 0$. For $x<0$, the only singularity in the lower half plane is the branch point at $w=-i k$. Hence we introduce a branch cut along the imaginary axis from $-i k$ to $-i \infty$ and choose a closing path of the variety we have just described for $0 \leq y \leq a, x>0$. Epon doing this we find that for $y<0$. $x<0$

$$
\phi(x, y)=\frac{i}{\pi} \int_{k}^{\infty} \frac{\exp (v x)}{T K_{-}(-i v)}\left[\frac{\gamma_{1} K}{(-i v-\kappa)}+\frac{(\kappa)}{(-i v+\kappa)}\right](n) T y d n .
$$

For $y<0, x>0$, we close above in a fashion similar to the case $x<0, y<0$ to obtain a function $\phi(x, y)$ which has the same functional form as we did for the region $x>0$, $0<y<a$. This incidently establishes the continuity of $\phi(x, y)$ for $x>0, y=0$.

VII. Remarks. We have now formulae for the region $x<0,0<y<a$, for $x>0$, and for $x<0, y<0$. For each of these expressions the integral or infinite sum, as the case may be, is uniformly and absolutely convergent. Due to the presence of the faroring exponential when $x \neq 0$, we may carry differentiation under the integral sign (or inside the summation). It is possible, therefore, to verify directly that the Eq. (2.1) and the boundary conditions $\left(2.1^{\prime}\right),\left(2.1^{\prime \prime}\right)$ appropriate to the region are satisfied. For $x<0$, $0<y<a$, we note that the expansion coefficients in the series are $0\left(n^{-3 / 2}\right)$ for $n \rightarrow \infty$. In view of the fact that we have decaying exponentials in this sum, it is clear that the asymptotic requirements of the region are met.

Consider the integral in the representation for $\phi$ when $x>0$. We write its integrand as $[\exp v x][h(y, v)]$. Now $h(y, v)$ is non-singular in $(-\infty,-k)$ and $0\left(v^{-3 / 2}\right)$ independent of $y$ as $v \rightarrow-\infty$. It follows $e^{r x}$ and $h(y, v)$ are $L^{2}(-\infty,-k)$ integrable and we have by Schwarz's inequity

$$
\begin{aligned}
\left|\int_{-k}^{-\infty} e^{v x} h(y, v) d v\right| & \leq\left[\int_{-k}^{-\infty} e^{2 x v} d v \int_{-k}^{-\infty}|h(y, v)|^{2} d v\right]^{1 / 2} \\
& \leq A\left[\int_{-\infty}^{-k} e^{2 x v} d v\right]^{1 / 2} \rightarrow 0 \text { as } x \rightarrow \infty \text { independently of } y .
\end{aligned}
$$

This shows the asymptotic requirements far to the right of the barrier are met.

The Riemann-Lebesque lemma shows the integral in the representations for $\phi$ when $x<0$ or when $x>0$ goes to zero as $y$ becomes negatively infinite. Since this is true of the exponential terms in the case $x>0$, we see that $\phi$ decays with depth. Clearly then, all of the order conditions we have had to assume in the formulation of the representations of $\phi(x, y)$ for $R_{1}$ and $R_{2}$ are satisfied.

VIII. Reflection and Transmission Coeffcients. From the results which we derived in Sec. VI, we may supply the refection and transmission coefficients of the traveling 
surface wares for $y=a, x \gtrless 0$. We note that for $x \rightarrow \infty$

$$
\phi(x, y)=\left[\gamma_{3} \exp (i \sigma x)+\gamma_{1} \exp (-i \sigma \cdot x)\right] \exp (\beta y)
$$

and this in turm is equal to

$$
\begin{aligned}
& -\left[\frac{\gamma_{1} K_{+}(\kappa)}{\sigma-\kappa}+\frac{\gamma_{2} K_{+}(-\kappa)}{\sigma+\kappa}\right] \frac{\beta^{2}}{\sigma} \frac{\exp (\beta)(y-2 a)}{K} \exp (i \sigma x) \\
+ & {\left[\frac{\gamma_{1} K \cdot(\kappa)}{-(\sigma+\kappa)}+\frac{\gamma_{2} K_{+}(-\kappa)}{\kappa-\sigma}\right] \frac{\beta^{2}}{\sigma} \frac{\exp )}{K(y-2 a)} \exp (-i \sigma x) . }
\end{aligned}
$$

From this we have then

$$
\gamma_{3}=-\left[\frac{\gamma_{1} K_{+}(\kappa)}{\sigma-\kappa}+\frac{\gamma_{2} K_{+}(-\kappa)}{\sigma+\kappa}\right] \frac{\beta^{2}}{\sigma} \frac{\exp (-2 \beta a)}{K_{+}(\sigma)}
$$

and

$$
\gamma_{4}=-\left[\frac{\gamma_{1} K_{+}(\kappa)}{\sigma+\kappa}+\frac{\gamma_{2} K_{-}(-\kappa)}{\sigma-\kappa}\right] \frac{\beta^{2}}{\sigma} \frac{\exp (-2 \beta a)}{K_{-}(-\sigma)}
$$

That is, we have two linear relations between the amplitudes of the traveling waves in the regions $x>0$ and $x<0, y=a$. The conditions (8.1) and (8.2) are independent as one can readily see by evaluating the determinant of the system (8.1) and (8.2).

As usual we have a relation between the magnitudes of $\gamma_{1}, \gamma_{2}, \gamma_{3}$ and $\gamma_{4}$. We form the integral

$$
\int\left(\phi \phi_{n}^{*}-\phi^{*} \phi_{n}\right) d s^{\prime}
$$

which is equal to zero since $\phi$ is source free and sufficiently regular. This integral is evaluated over the "boundary" of the channel with due recognition for the barrier. In view of the particular asymptotic forms we have found for $\phi$ for $|x| \rightarrow \infty, v \rightarrow-\infty$ (8.3) reduces to

$$
\begin{aligned}
\left|\gamma_{3}\right|^{2}-\left|\gamma_{4}\right|^{2}=\left[\left|\gamma_{1}\right|^{2}-\left|\gamma_{2}\right|^{2}\right] & \\
& \cdot \frac{\beta a \kappa\left(\beta a-\beta^{2} a^{2}+\alpha_{0}^{2}\right)[\exp (-2 \beta a)]\left[1+\exp \left(-2 \alpha_{0}\right)\right]}{2 \sigma \alpha_{0}^{2}\left(\alpha_{0}-a \beta\right)} .
\end{aligned}
$$

The expressions we have found for $\gamma_{3}$ and $\gamma_{4}$ satisfy this relation. On the basis of (8.4) we can define the reflection coefficients and transmission coefficients. Note that if $\gamma_{4}=0$, we have transmission to the right and reflection to the left. $\left|\gamma_{2}\right|=\left|\gamma_{2} / \gamma_{1}\right|$ is the magnitude of the left reflection coefficient. Then

$$
\left|\frac{\gamma_{3}}{\gamma_{1}}\right|^{2} \frac{2 \sigma \alpha_{0}^{2}\left(\alpha_{0}-a \beta\right) \exp (2 a \beta)}{\beta a \kappa\left(\beta a-\beta^{2} a^{2}+\alpha_{0}^{2}\right)\left[1+\exp \left(-2 \alpha_{0}\right)\right]}
$$

defines the transmission coefficient to the right, that is $\left|t_{R}\right|$. We find in this case

$$
\begin{aligned}
& \left|r_{L}\right|^{2}=\left(\frac{\sigma-\kappa}{\sigma+\kappa}\right)^{2}, \\
& \left|t_{R}\right|^{2}=\frac{4 \sigma \kappa}{(\sigma+\kappa)^{2}} .
\end{aligned}
$$


Similarly $\left|r_{R}\right|=\left|r_{L}\right|$ and $\left|t_{L}\right|=\left|t_{R}\right|$, that is for the case in which $\gamma_{2}=0$. Note that for the case $k=0$

$$
\begin{aligned}
& \left|r_{L}\right|^{2}=\left|r_{R}\right|^{2}=\left(\frac{a \beta-\alpha_{0}}{a \beta+\alpha_{0}}\right)^{2}, \\
& \left|t_{L}\right|^{2}=\left|t_{R}\right|^{2}=\frac{4 a \beta \alpha_{0}}{\left(a \beta+\alpha_{n}\right)^{2}} .
\end{aligned}
$$

Had we only been interested in magnitudes of reflection and transmission coefficients, these results might have been anticipated from classical transmission line theory. However, the determination of the various reflection and transmission coefficients involves a phase for each of these coefficients. These angles may be calculated from $K_{+}(\pi)$, $K_{+}(-\kappa), K_{+}(\sigma)$ and $K_{+}(-\sigma)$ but we shall not pursue this point here.

\section{BIBLIOGRAPHY}

1. A. E. Heins, Water Waves over a Channel of Finile Depth with a Dock, American Journal of Mathematics LXX, 730-748 (1948).

2. A. E. Heins, Water Waves over a Channel of Finite Depth, Canadian Journal of Mathematics II, 210222 (1950).

3. A. E. Heins, Some Remarks on the Coupling of Two Ducts, Journal of Mathematics and Physics, XXX, 164-169 (1951).

4. J. J. Stoker, Surface Waves in Water of Variable Depth, Q. of Appl. Math., 5, 1-54 (1947).

5. R. E. A. C. Paley and N. Wiener, The Fourier Transform in the Complex Domain, American Mathematical Society Colloquium Publication, 1934, Chapter IV.

6. E. C. Titchmarsh, Introduction to the Theory of the Fourier Integral, Oxford 1948, p. 15, Theorem 40. 